\title{
Development of a conceptual model of sustainable building from a university
}

\author{
Alexandru Olteanu ${ }^{l, *}$ and Claudiu-Vasile Kifor $^{l}$ \\ ${ }^{1}$ Lucian Blaga University of Sibiu. Faculty of Engineering, Industrial Engineering and Management \\ Department, Str. Emil Cioran, Nr.4, Sibiu, 550025, Romania
}

\begin{abstract}
Introduction: In this paper, the authors aim is to develop a conceptual model that aims to facilitate the processes of implementing sustainability and / or assessment of sustainable performance in a Higher Education Building (HEB). The conceptual model could also be used to compare two or more buildings of higher education in terms of sustainability. Methodology: The authors started the scientific research with the literature review in order to identify and analyze the most relevant aspects that are frequently assess and could influencing the components of sustainability in higher education institutions. These aspects were clustered into categories and for each of them were established objectives and key performance indicators. Results: The main results obtained were presented synthetically in graphical, tabular and mathematical manner. This way of presentation was preferred by the authors for defining and developing the proposed conceptual model. Conclusions: Finally, the authors propose a piloting of the conceptual model in order to validate it, because until now, no testing in physical circumstances of the model has been implemented, it based only on the results identified in the literature review.
\end{abstract}

\section{Introduction}

The aim of this paper is to develop a conceptual model that aims to facilitate the processes of implementing sustainability in a Higher University Building (HEB) and/or assessment it's sustainable development performance. The conceptual model can also be used to compare two or more HEBs in terms of sustainability. The paper begins with the definition of the main concepts used, as follows:

Sustainability - is the ability of a macro or micro economic entity to ensure it's resources in the long term, without affecting the development of future generations, based on three essential environments: the social environment, the economic environment and the environment $[1,2,3$, $4,5,6,7]$.

Sustainable University - is a higher education institution that educates word wide citizens for sustainable development, with a great social responsibility, to raise awareness and to inoculate knowledge, as well as to develop and implement technologies and tools necessary for a sustainable future. At the same time, it provides relevant information on urgent societal challenges and is committed to reducing social and environmental footprints, encouraging

*Corresponding author: alex.olteanu@ulbsibiu.ro 
stakeholders to participate actively and responsibly, making sustainability a central priority for both it and society $[8,9]$.

Building's key performance indicators - by measuring performance with key performance indicators (KPIs) gives building managers a clear view of the targets they need to meet to achieve efficient building energy consumption. For example, electricity, gas and water consumption per square meter, per person [10]. To formulate the calculation process of KPIs, formula or equations are always used. A well-defined KPI provides a quantitative metric to compare different buildings [11].

University ranking - a transparent tool of fair university evaluation for the public, which may have a huge impact on higher education institution decision making [12].

In the next stage, a series of national and international rankings were identified and analyzed that evaluate the activity of universities in terms of sustainability, with an emphasis on the environmental component. To this end, five rankings in the field of university sustainability have been identified. Of these, two rankings evaluate universities around the world, one ranking evaluates universities in the UK, and a ranking and a system for tracking the sustainability of US universities.

UI Green Metric World University Ranking (UIGM) [13]: is an initiative of University of Indonesia which is launched in 2010 , to which a number of experts in worldwide university rankings have been invited to discuss. Following the discussions, it was concluded that the current criteria used for ranking universities do not give credit to those universities that strive to reduce their carbon footprint. Thus, it was noted the need for a uniform system that is suitable for worldwide universities and in which the results are based on a numerical score that allows ranking, so that they can make quick comparisons between them on the criteria of commitment to solve the problems of sustainability and environmental impact. The aim of this ranking is to provide an overview of the current state and policies related to the green campus and sustainability in universities around the world. It is expected that, drawing the attention of university leaders and stakeholders, more attention will be paid to combating global climate change, conserving energy and water, recycling waste and green transport. All this requires changing behaviour and paying greater attention to environmental sustainability, as well as economic and social issues related to sustainability.

The Times Higher Education World University Rankings (THE WUR) [14]: are the only global performance tables that assess universities against the United Nations' Sustainable Development Goals (SDGs) using carefully calibrated indicators to provide comprehensive and balanced comparisons across four broad areas: research, stewardship, outreach and teaching. The last edition includes 768 universities from 85 countries. There are 17 UN SDGs and this ranking evaluates the specific performance of each of them. Thus, there is a specific evaluation methodology for each SDG.

People \& Planet University League (P\&PUL) [15]: is the only comprehensive and independent ranking of UK universities ranked by environmental and ethical performance. The criteria assessed by this ranking are presented in figure 1 .

Best Colleges (BC) [16]: is a US ranking that evaluates among others the USA Greenest Universities. In this regard, Best Colleges combined their scoring system, which measures academic quality, with STARS ratings which identifying the best U.S. colleges and universities that have also distinguished as the nation's greenest universities. The goal of this ranking is to spotlight schools that have launched the most impactful initiatives to reduce on-campus waste and energy consumption, promote alternative transportation, provide funding to student and faculty-led green proposals, and take other measures for the environment benefit. 


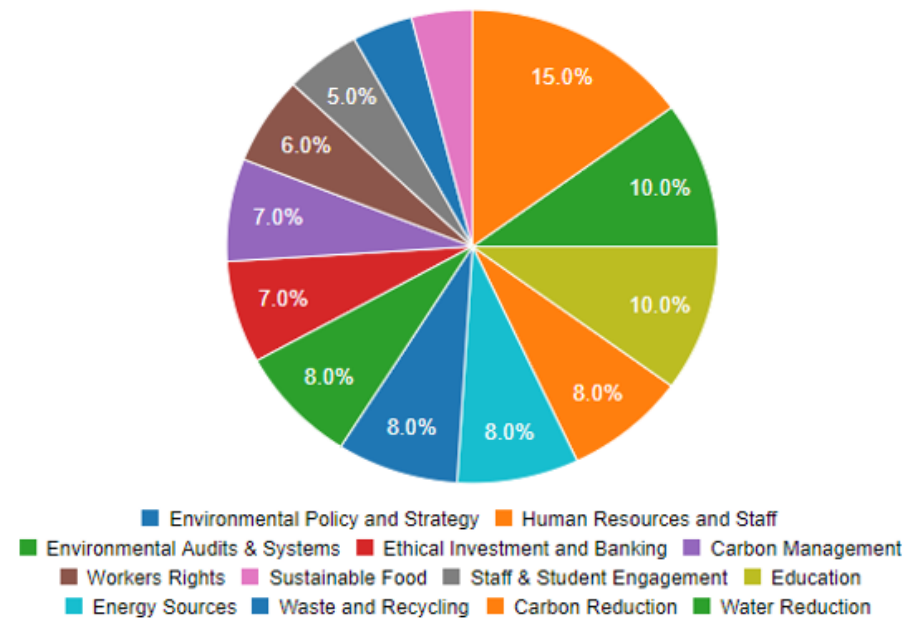

Fig. 1. Assessment criteria [15]

The Sustainability Tracking, Assessment \& Rating System (STARS) [17]: is a transparent, self-reporting framework for colleges and universities to measure their sustainability performance. The framework encompasses long-term sustainability goals for already highachieving institutions, as well as entry points of recognition for institutions that are taking first steps toward sustainability. STARS is designed to provide a framework for understanding sustainability in all sectors of higher education, creating incentives for continual improvement toward sustainability and for build a stronger, more diverse campus sustainability community.

In addition to the rankings presented above, an article was added that it made a European rating system for sustainable student housing. Also the specific legislation and the regulations on public buildings has been revised. One of the most important laws in this regard is Directive 2010/31 / EU, amended in 2018 by Directive (EU) 2018/844, on the energy performance of buildings [18]. Its aim is to improve the energy performance of buildings in the EU, taking into account different climatic and local conditions and to accelerate the costeffective renovation of existing buildings and to promote smart technologies in buildings [18]. Directive 2018/844 of the European Parliament and of the Council of 30 May 2018 request among other that "The Union is committed to developing a sustainable, competitive, secure and decarbonised energy system. The Energy Union and the Energy and Climate Policy Framework for 2030 establish ambitious Union commitments to reduce greenhouse gas emissions further by at least $40 \%$ by 2030 as compared with 1990, to increase the proportion of renewable energy consumed" [19]. Subsequently, the European Parliament voted for a more ambitious target of a $60 \%$ reduction in greenhouse gases by 2030 [20]. There are also a number of laws, standards and concepts that refer to energy efficiency and reducing carbon emissions $[21,22,23,24,25,26,27]$, minimization the amount of waste generated [28, 29] or accessibility for disabilities people [30] that have been taken into account for the design of KPIs.

From the five rankings identified, the most important criteria for assessing sustainability were extracted. Thus, new groups were formed with most important assessment criteria of all identified rankings and with some new ones developed by the authors based on the identified ones. Another aspect that was taken into account was the achievement some of the sustainable development goals that are strongly related to student quality of life in the 2030 Agenda for Sustainable Development. Among them can be listed: good health and well-being (Goal 3), quality of education (Goal 4), affordable and clean energy (Goal 7), sustainable cities and communities (Goal 11) and climate action (Goal 13) [31]. 


\section{Methodology}

Following the review of the specialized literature, a qualitative analysis in order to selection of the most relevant and current sources was performed. The information collected was then analyzed and presented in tabular form. Thus, the information was grouped into categories and for each of them objectives and key performance indicators were develop. The tabular structure helps to a better observe and understand the data. Thus, the tabular clustering was performed by categories and for each assesst criterion identified, objectives and key performance indicators were developed. The tabular structure helps to better observe and understand the data. At the same time, the data presented in the table were subjected to a qualitative analysis to select the most relevant assessment criteria for the to the risearch topic. The qualitative analysis was based on a brainstorming session with specialists in interest fields from the Faculty of Engineering.

In the next stage, an quantitative analysis was performed that contributed to the development of the mathematical model. Mathematical modeling represents the properties of the conceptual model proposed to facilitate the processes of implementing sustainability or assessment the sustainability performance of a Higher Education Building (HEB).

\section{Results}

Following the data collection, a synthesis of the identified relevant assessment criteria in the studied rankings was performed. Subsequently, they were divided into three main categories (modules): Building Design and Management (BDM); Environmental Protection and Quality of the indoor (EPQ); Human Resource and other Stakeholders (HRS); and ten secondary categories, presented in tables 3 - 5. Also, in figure 2 you can see the distribution of the ten subcategories according to the main categories.

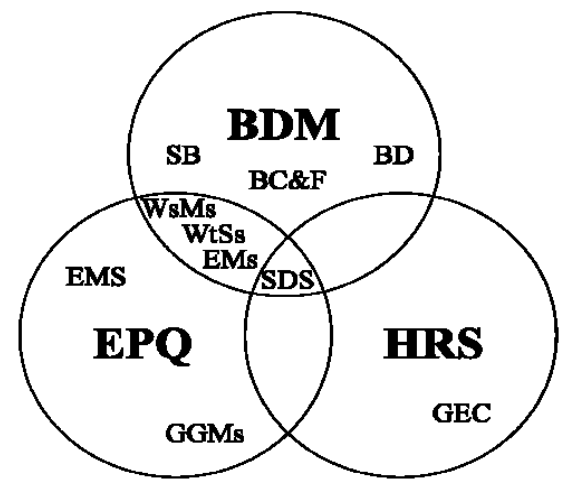

Fig. 2 Categories intersection

Thus, in figure 2 it can be seen that a single subcategory (SDS) falls in all 3 main categories and 3 subcategories (WsMs; WtSs; EMs) falls in two of them.

At the same time, table 1 shows how the main categories were divided according to the three pillars of sustainability, in order to observe the distribution of the 10 subcategories according to them.Thus it can be seen that the SDS subcategory falls all the analyzed categories. 
Table 1 Subcategories

\begin{tabular}{|c|c|c|c|c|c|c|c|c|c|c|}
\hline \multirow{2}{*}{ No } & \multicolumn{2}{|r|}{ Subcategories } & \multicolumn{3}{|c|}{ BDM } & \multicolumn{3}{|c|}{ EPQ } & \multicolumn{2}{|c|}{ HRS } \\
\hline & Code & Name & & & So & & Ec & So & & Ec|So \\
\hline 1 & SB & Smart Building & & & $\checkmark$ & & & & & \\
\hline 2 & BD & Building Design & $\checkmark$ & $\sqrt{ }$ & $\checkmark$ & & & & & \\
\hline 3 & $\mathrm{BC} \& \mathrm{~F}$ & Building Comfort \& Facilities & $\checkmark$ & $\checkmark$ & $\checkmark$ & & & & & \\
\hline 4 & EMS & Environmental Management System & & & & $\checkmark$ & $\checkmark$ & $\checkmark$ & & \\
\hline 5 & EMs & Energy Efficiency system & $\checkmark$ & $\checkmark$ & $\checkmark$ & $\checkmark$ & $\checkmark$ & $\sqrt{ }$ & & \\
\hline 6 & GGMs & $\begin{array}{c}\text { Greenhouse Gasses Management } \\
\text { system }\end{array}$ & & & & $\checkmark$ & $\checkmark$ & $\checkmark$ & & \\
\hline 7 & WsMs & Waste Management system & $\checkmark$ & $\checkmark$ & $\checkmark$ & $\checkmark$ & $\checkmark$ & $\checkmark$ & & \\
\hline 8 & WtSs & Water Management system & $\checkmark$ & $\checkmark$ & $\checkmark$ & $\sqrt{ }$ & $\checkmark$ & $\checkmark$ & & \\
\hline 9 & GEC & Gender Equality and Chance & & & & & & & & $\checkmark \mid \checkmark$ \\
\hline 10 & SDS & Sustainable Development Strategy & $\checkmark$ & $\checkmark$ & $\checkmark$ & $\checkmark$ & $\checkmark$ & $\checkmark$ & \begin{tabular}{l|l} 
& $\checkmark$ \\
\end{tabular} & \begin{tabular}{l|l}
$\checkmark$ & $\checkmark$ \\
\end{tabular} \\
\hline
\end{tabular}

Also, from the 6 main sources analyzed, a total of 85 sustainability assessment criteria were obtained, being subsequently adapted for the evaluation of a HEB. In table 2 a quantitative analysis was performed where the number of identified criteria for each main category can be seen. Thus, the distribution of the identified criteria on the three pillars of sustainability according to their specificity was presented quantitatively. The criteria distribution (table 2) was made: separately - on each type of pillar; combined - on two pillars; and simultaneously all three pillars of sustainability.

Table 2 Quantitative analysis of assess criteria

\begin{tabular}{|c|c|c|c|}
\hline \multirow{2}{*}{$\begin{array}{c}\text { Sustainability } \\
\text { pillars }\end{array}$} & \multicolumn{3}{|c|}{ Assess criteria per categories } \\
\cline { 2 - 4 } & BDM & EPQ & HRS \\
\hline En & 7 & 4 & - \\
\hline Ec & - & - & - \\
\hline So & - & 6 & 12 \\
\hline En+Ec & 9 & 2 & - \\
\hline En+So & 6 & - & 2 \\
\hline Ec+So & 3 & - & 5 \\
\hline En+Ec+So & 8 & 4 & 17 \\
\hline Total & $\mathbf{3 3}$ & $\mathbf{1 6}$ & $\mathbf{3 6}$ \\
\hline
\end{tabular}

It is ideal for each category of criteria to achieve all three pillars of sustainability in order to ensure a complete and effective assessment. It can be seen in table 2 that there are criteria that fall into two or even all three pillars of sustainability. Therefore each module can make an independent assessment of the HEB sustainability. This is a major advantage of the model because it can independently assess the sustainability performance of the building, environmental protection or human resource.

In this sense, for each identified evaluation criterion, KPIs were created for the calculation and evaluation of the sustainability of a higher education building (HEB), presented in tables 35 Some of the KPIs were made on the basis of standards, articles and legislation analyzed. These KPIs will be calculated in the range [0-1] and each of them should tend towards the ideal, where 1 is considered ideal. 
Table 3 Building design and management key performance indicators

\begin{tabular}{|c|c|c|c|c|c|}
\hline \multirow{3}{*}{ No. } & \multirow{2}{*}{\multicolumn{2}{|c|}{ Category }} & \multicolumn{3}{|c|}{ Key Performance Indicators } \\
\hline & & & \multicolumn{3}{|c|}{ Building Design and Management (BDM) } \\
\hline & Code & Goals & Environmental KPI & \begin{tabular}{|c|} 
Economic KPI \\
\end{tabular} & Social KPI \\
\hline 1 & SDS & $\begin{array}{l}\text { Sustainable Building Strategy } \\
{[15]}\end{array}$ & \multicolumn{3}{|c|}{$\begin{array}{c}\text { SBS }=1 \\
\text { where: } \text { SBS }=\text { Sustainable Building Strategy }\end{array}$} \\
\hline 2 & $\mathrm{BD}$ & HEB Design $[17,16]$ & $\begin{array}{c}\mathrm{SEW} / \mathrm{TW}=1 \\
\text { where: } \mathrm{SEW}=\text { South-Est Windows } \\
\text { orientation } \\
\mathrm{TW}=\text { Total Windows orientations [23] }\end{array}$ & \begin{tabular}{|c|}
$\mathrm{SEW} / \mathrm{TW}=1$ \\
where: $\mathrm{SEW}=$ South-Est Windows \\
orientation \\
$\mathrm{TW}=$ Total Windows orientations [23]
\end{tabular} & $\mathrm{x}$ \\
\hline 3 & $\mathrm{BD}$ & HEB Construction $[17,16]$ & $\begin{array}{c}\text { VEM/VTM=1 } \\
\text { where: } \mathrm{VEM}=\text { Volume of Ecological } \\
\text { Materials } \\
\text { VTM= Volume of Total Materials } \\
{[21,20]}\end{array}$ & 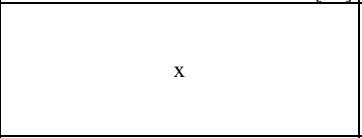 & $\mathrm{x}$ \\
\hline 4 & $\mathrm{BD}$ & $\begin{array}{l}\text { Elements of green building } \\
\text { implementation as reflected in all } \\
\text { construction and renovation } \\
\text { policy of HEB [13] }\end{array}$ & $\begin{array}{c}\mathrm{VEM} / \mathrm{VTM}=1 \\
\text { where: } \mathrm{VEM}=\mathrm{V} \text { olume of ecological } \\
\text { materials } \\
\text { VEM=Volume of total materials }[21, \\
20]\end{array}$ & $\mathrm{x}$ & $\mathrm{x}$ \\
\hline 5 & $\mathrm{BD}$ & $\begin{array}{l}\text { Class space/total class students } \\
\qquad[31]\end{array}$ & $\begin{array}{c}1 \mathrm{SS}=3 \mathrm{~m} 2 \\
\text { where: OSS = One Student ideal } \\
\text { Surface; } \\
3 \mathrm{~m} 2=1[32]\end{array}$ & $\mathrm{x}$ & $\mathrm{x}$ \\
\hline 6 & SB & $\begin{array}{c}\text { HEB operations and maintenance } \\
\text { (SMART } \\
{[17,16]}\end{array}$ & 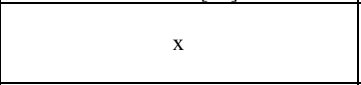 & $\begin{array}{c}\mathrm{AP} / \mathrm{TP}=1 \\
\text { where: } \mathrm{AP}=\text { Automated Processes } \\
\mathrm{TP}=\text { Total Processes }\end{array}$ & $\begin{array}{c}\mathrm{AP} / \mathrm{TP}=1 \\
\text { where: } \mathrm{AP}=\text { Automated Processes } \\
\mathrm{TP}=\text { Total Processes }\end{array}$ \\
\hline 7 & SB & $\begin{array}{l}\text { Smart building implementation } \\
\qquad[13]\end{array}$ & $\mathrm{x}$ & $\begin{array}{c}\mathrm{AP} / \mathrm{TP}=1 \\
\text { where: } \\
\mathrm{AP}=\text { Automated Processes; } \\
\mathrm{TP}=\text { Total Processes }\end{array}$ & $\begin{array}{c}\mathrm{AP} / \mathrm{TP}=1 \\
\text { where: } \\
\mathrm{AP}=\text { Automated Processes; } \\
\mathrm{TP}=\text { Total Processes }\end{array}$ \\
\hline 8 & $\mathrm{BC} \& \mathrm{~F}$ & Inclusive Access & $\mathrm{x}$ & $\mathrm{x}$ & $\begin{array}{c}\mathrm{RA} / \mathrm{TA} 1=1 \\
\text { where: } \mathrm{RA}=\text { Ramp Access }\end{array}$ \\
\hline
\end{tabular}




\begin{tabular}{|c|c|c|c|c|c|}
\hline \multirow{3}{*}{ No. } & \multirow{2}{*}{\multicolumn{2}{|c|}{ Category }} & \multicolumn{3}{|c|}{ Key Performance Indicators } \\
\hline & & & \multicolumn{3}{|c|}{ Building Design and Management (BDM) } \\
\hline & Code & Goals & Environmental KPI & \begin{tabular}{|c|} 
Economic KPI \\
\end{tabular} & Social KPI \\
\hline & & & & & $\mathrm{TA}=$ Total Access $[30]$ \\
\hline 9 & $B C \& F$ & $\begin{array}{c}\text { Source of natural ventilation in all } \\
\text { classrooms and laboratories [31] }\end{array}$ & $\begin{array}{c}\mathrm{SNV}=1 \\
\text { where: } \mathrm{NV}=\text { Source Natural } \\
\text { Ventilation [33] }\end{array}$ & $\mathrm{x}$ & $\begin{array}{c}\text { SNV }=1 \\
\text { where: } \mathrm{NV}=\text { Source Natural } \\
\text { Ventilation }\end{array}$ \\
\hline 10 & $\mathrm{BC} \& \mathrm{~F}$ & $\begin{array}{c}\text { Mechanical ventilation in all } \\
\text { classrooms and laboratories [31] }\end{array}$ & (1) & $\begin{array}{c}\text { SMV }=1 \\
\text { where: NV = Source Mechanical } \\
\text { Ventilation [33] }\end{array}$ & $\begin{array}{c}\text { SMV }=1 \\
\text { where: } \mathrm{NV}=\text { Source Mechanical } \\
\text { Ventilation [33] }\end{array}$ \\
\hline 11 & $\mathrm{BC} \& \mathrm{~F}$ & $\begin{array}{l}\text { The optimal amount of light in all } \\
\text { classrooms and laboratories [31] }\end{array}$ & $\begin{array}{c}\mathrm{OAL}=300 \text { lux }[33][34] \\
\text { where: } 300 \text { lux }=1 ; \\
\mathrm{OAL}=\text { Optimal Amount of Light }\end{array}$ & $\begin{array}{c}\mathrm{OAL}=300 \text { lux }[33][34] \\
\text { where: } 300 \text { lux }=1 ; \\
\mathrm{OAL}=\text { Optimal Amount of Light }\end{array}$ & $\begin{array}{c}\mathrm{OAL}=300 \text { lux }[33][34] \\
\text { where: } 300 \text { lux }=1 ; \\
\mathrm{OAL}=\text { Optimal Amount of Light }\end{array}$ \\
\hline 12 & $\mathrm{BC} \& \mathrm{~F}$ & Thermal insulated building [31] & $\begin{array}{c}\text { TI = 1 } \\
\text { where: } \mathrm{TI}=\text { Thermal Insulate [33] }\end{array}$ & $\begin{array}{c}\mathrm{TI}=1 \\
\text { where: } \mathrm{TI}=\text { Thermal Insulate [33] }\end{array}$ & $\mathrm{x}$ \\
\hline 13 & $\mathrm{BC} \& \mathrm{~F}$ & $\begin{array}{l}\text { Building thermal comfort in all } \\
\text { classrooms and laboratories [31] }\end{array}$ & $\begin{array}{c}\mathrm{Tc}=20-26^{\circ} \mathrm{C} \\
\text { where: } 20-26^{\circ} \mathrm{C}=1 \\
\mathrm{TC}=\text { Thermal comfort }[33]\end{array}$ & $\begin{array}{c}\mathrm{Tc}=20-26^{\circ} \mathrm{C} \\
\text { where: } 20-26^{\circ} \mathrm{C}=1 \\
\mathrm{TC}=\text { Thermal comfort }[33]\end{array}$ & $\begin{array}{c}\mathrm{Tc}=20-26^{\circ} \mathrm{C} \\
\text { where: } 20-26^{\circ} \mathrm{C}=1 ; \\
\mathrm{TC}=\text { Thermal comfort }[33]\end{array}$ \\
\hline 14 & $\mathrm{BC} \& \mathrm{~F}$ & Acoustic insulated building [31] & $\begin{array}{c}\mathrm{AI}=1 \\
\text { where: } \mathrm{AI}=\text { Acoustic Insulate [33] }\end{array}$ & $\begin{array}{c}\mathrm{AI}=1 \\
\text { where: } \mathrm{AI}=\text { Acoustic Insulate [33] }\end{array}$ & ( \\
\hline 15 & $\mathrm{BC} \& \mathrm{~F}$ & $\begin{array}{c}\text { Reverberation time in all } \\
\text { classrooms and laboratories [31] }\end{array}$ & $\begin{array}{c}\mathrm{Rt}=0.5 \mathrm{~s} \pm 20 \% \\
\text { where: } 0.5 \mathrm{~s} \pm 20 \%=1 \\
\mathrm{Rt}=\text { Reverberation time }[33]\end{array}$ & ( & $\begin{array}{c}\mathrm{Rt}=0.5 \mathrm{~s} \pm 20 \% \\
\text { where: } 0.5 \mathrm{~s} \pm 20 \%=1 \\
\mathrm{Rt}=\text { Reverberation time [33] }\end{array}$ \\
\hline 16 & $\mathrm{BC} \& \mathrm{~F}$ & $\begin{array}{c}\text { Humidity level in all classrooms } \\
\text { and laboratories [31] }\end{array}$ & $\begin{array}{c}\mathrm{Hl}=30-50 \% \\
\text { where: } 30-50 \%=1 ; \\
\mathrm{Hl}=\text { Humidity level }[33]\end{array}$ & $\mathrm{x}$ & $\begin{array}{c}\mathrm{Hl}=30-50 \% \\
\text { where: } 30-50 \%=1 ; \\
\mathrm{Hl}=\text { Humidity level }[33]\end{array}$ \\
\hline 17 & EMs & Energy Management system [31] & \multicolumn{3}{|c|}{$\begin{array}{c}\text { EMS=1 } \\
\text { where: } \text { EMS }=\text { Energy Management System }\end{array}$} \\
\hline 18 & EMs & $\begin{array}{l}\text { Energy efficient installations and } \\
\text { systems usage in HEB are } \\
\text { replacing conventional } \\
\text { installations and systems [13] }\end{array}$ & $\begin{array}{c}\text { EEIS/TIS }=1 \\
\text { where: } \\
\text { EEIS = Energy Efficient Installations } \\
\text { and Systems; } \\
\text { TIS = Total Installations and Systems } \\
{[21,22,23,24,25,26,27]}\end{array}$ & \begin{tabular}{|c|} 
EEIS/TIS $=1$ \\
where: \\
EEIS = Energy Efficient Installations \\
and Systems; \\
TIS = Total Installations and Systems \\
{$[21,22,23,24,25,26,27]$}
\end{tabular} & $\mathrm{x}$ \\
\hline
\end{tabular}




\begin{tabular}{|c|c|c|c|c|c|}
\hline \multirow{3}{*}{ No. } & \multirow{2}{*}{\multicolumn{2}{|c|}{ Category }} & \multicolumn{3}{|c|}{ Key Performance Indicators } \\
\hline & & & \multicolumn{3}{|c|}{ Building Design and Management (BDM) } \\
\hline & Code & Goals & Environmental KPI & \begin{tabular}{|c|} 
Economic KPI \\
\end{tabular} & Social KPI \\
\hline 19 & EMs & $\begin{array}{c}\text { Energy efficient appliances usage } \\
\text { in HEB are replacing } \\
\text { conventional appliances [13] }\end{array}$ & $\begin{array}{c}\text { EEA/TA }=1 \\
\text { where: Energy efficient } \\
\text { appliances/Total appliances }\end{array}$ & $\begin{array}{c}\mathrm{EEA} / \mathrm{TA}=1 \\
\text { where: Energy efficient } \\
\text { appliances/Total appliances }\end{array}$ & $\mathrm{x}$ \\
\hline 20 & EMs & $\begin{array}{c}\text { Energy efficiency (HEB Total) } \\
{[14,13,16,17]}\end{array}$ & $\begin{array}{c}60(\mathrm{kWh} / \mathrm{m} 2 / \mathrm{a}) / \mathrm{TEU}(\mathrm{kWh} / \mathrm{m} 2 / \mathrm{an})=1 \\
\text { where: TEU }=\text { Total Energy Usage } \\
\\
{[22]}\end{array}$ & $\begin{array}{c}60(\mathrm{kWh} / \mathrm{m} 2 / \mathrm{a}) / \mathrm{TEU}(\mathrm{kWh} / \mathrm{m} 2 / \mathrm{an})=1 \\
\text { where: } \mathrm{TEU}= \\
\text { Total Energy Usage } \\
{[22]}\end{array}$ & $\mathrm{x}$ \\
\hline 21 & EMs & $\begin{array}{l}\text { HEB heating energy efficiency } \\
\qquad[17,16,14]\end{array}$ & 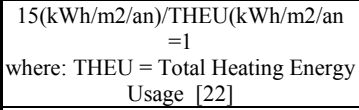 & $\begin{array}{c}\begin{array}{c}15(\mathrm{kWh} / \mathrm{m} 2 / \mathrm{an}) / \mathrm{THEU}(\mathrm{kWh} / \mathrm{m} 2 / \mathrm{an} \\
=1 \\
\text { where: THEU }=\text { Total Heating Energy } \\
\text { Usage [22] }\end{array} \\
\end{array}$ & $\mathrm{x}$ \\
\hline 22 & EMs & $\begin{array}{l}\text { HEB cooling energy efficiency } \\
{[117,16,14]}\end{array}$ & $\begin{array}{c}15(\mathrm{kWh} / \mathrm{m} 2 / \mathrm{an}) / \mathrm{TCEU}(\mathrm{kWh} / \mathrm{m} 2 / \mathrm{an}= \\
1 \\
\text { where: TCEU }=\text { Total Cooling Energy } \\
\text { Usage [22] }\end{array}$ & $\begin{array}{c}15(\mathrm{kWh} / \mathrm{m} 2 / \mathrm{an}) / \mathrm{TCEU}(\mathrm{kWh} / \mathrm{m} 2 / \mathrm{an}= \\
1 \\
\text { where: TCEU }=\text { Total Cooling Energy } \\
\text { Usage }[22]\end{array}$ & $\mathrm{x}$ \\
\hline 23 & WsMs & Waste Management system & \multicolumn{3}{|c|}{$\begin{array}{c}\text { WsMS }=1 \\
\text { WsMS }=\text { Waste Management System }\end{array}$} \\
\hline 24 & WsMs & Organic waste treatment [13] & $\begin{array}{c}\mathrm{TOW} / \mathrm{OW}=1 \\
\text { where: } \mathrm{TOW}=\text { Treated Organic } \\
\text { Waste; } \\
\text { OW = Organic Waste }[28,29]\end{array}$ & ( & $\begin{array}{c}\text { TOW/OW }=1 \\
\text { where: } \text { TOW = Treated Organic } \\
\text { Waste; } \\
\text { OW = Organic Waste }[28,29]\end{array}$ \\
\hline 25 & WsMs & Toxic waste handled [13] & $\begin{array}{c}\text { TxWH/TW }=1 \\
\text { where: } \text { TxWH = Toxic Waste } \\
\text { Handled; } \\
\text { TxW }=\text { Toxic Waste }[28,29]\end{array}$ & $\mathrm{x}$ & $\begin{array}{c}\text { TxWH/TW }=1 \\
\text { where: TxWH = Toxic Waste } \\
\text { Handled; } \\
\text { TxW = Toxic Waste }[28,29]\end{array}$ \\
\hline 26 & WsMs & $\begin{array}{l}\text { Proportion of recycling and } \\
\text { reusing HEB waste } \\
{[15,13,14]}\end{array}$ & $\begin{array}{c}\mathrm{R} \& \mathrm{RW} / \mathrm{TW}=1 \\
\text { where: } \mathrm{R} \& \mathrm{RW}=\text { Recycling and } \\
\text { Reusing Waste; } \\
\mathrm{TW}=\text { total waste }[28,29]\end{array}$ & $\mathrm{x}$ & 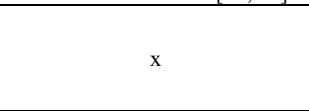 \\
\hline 27 & WsMs & $\begin{array}{c}\text { HEB waste minimization }[17,16, \\
15,13]\end{array}$ & $\begin{array}{c}\text { NMW/CMW } \approx 0(0=1)^{*} \\
\text { where: NMW = Next Month Waste; } \\
\text { CMW = Crrent Month Wast }[28,29]\end{array}$ & $\mathrm{x}$ & $\mathrm{x}$ \\
\hline
\end{tabular}




\begin{tabular}{|c|c|c|c|c|c|}
\hline \multirow{3}{*}{ No. } & \multirow{2}{*}{\multicolumn{2}{|c|}{ Category }} & \multicolumn{3}{|c|}{ Key Performance Indicators } \\
\hline & & & \multicolumn{3}{|c|}{ Building Design and Management (BDM) } \\
\hline & Code & Goals & Environmental KPI & Economic KPI & Social KPI \\
\hline 28 & WsMs & $\begin{array}{c}\text { Construction and demolition } \\
\text { waste recycling at source } \\
{[17,16]}\end{array}$ & $\begin{array}{c}\text { RC\&DW/TC\&DW }=1 \\
\text { where: } \text { RC\&DW }=\text { Construction and } \\
\text { Demolition Recycled Waste; } \\
\text { TC\&DW = Total Construction and } \\
\text { Demolition Waste }[28,29]\end{array}$ & $\mathrm{x}$ & $\mathrm{x}$ \\
\hline 29 & WtMs & Water Management System & \multicolumn{3}{|c|}{$\begin{aligned} \text { WtMS }=1 \\
\text { where: } \text { WtMS }=\text { Water Management System }\end{aligned}$} \\
\hline 30 & WtMs & $\begin{array}{c}\text { The use of water efficient } \\
\text { appliances (water tap, toilet flush, } \\
\text { etc.) [13] }\end{array}$ & $\begin{array}{c}\text { WEA/TWA }=1 \\
\text { where: WEA = Water Efficient } \\
\text { Appliances; } \\
\text { TWA = Total Water Appliances }\end{array}$ & $\begin{array}{c}\text { WEA/TWA =1 } \\
\text { where: WEA = Water Efficient } \\
\text { Appliances; } \\
\text { TWA = Total Water Appliances }\end{array}$ & $\mathrm{x}$ \\
\hline 31 & WtMs & $\begin{array}{c}\text { Proportion of reused and recycled } \\
\text { water }[14,13]\end{array}$ & $\begin{array}{c}\mathrm{R} \& \mathrm{RW} / \mathrm{TWU}=1 \\
\text { where: } \mathrm{R} \& \mathrm{RW}=\text { Reused or Recycled } \\
\text { Water } \\
\text { TWU }=\text { Total Water Usage }\end{array}$ & 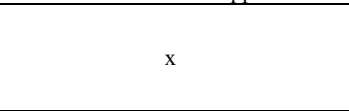 & $\begin{array}{c}\mathrm{R} \& \mathrm{RW} / \mathrm{TWU}=1 \\
\text { where: } \mathrm{R} \& \mathrm{RW}=\text { Reused or Recycled } \\
\text { Water } \\
\mathrm{TWU}=\text { Total Water Usage }\end{array}$ \\
\hline 32 & WtMs & Gray water reusing $[17,16]$ & $\begin{array}{c}\text { GWR/TWW }=1 \\
\text { where: GWP = Gray Water Reusing; } \\
\text { TWW = Total Water Washbasin }\end{array}$ & $\begin{array}{c}\text { GWR/TWW }=1 \\
\text { where: GWP = Gray Water Reusing; } \\
\text { TWW = Total Water Washbasin }\end{array}$ & $\begin{array}{c}\text { GWR/TWW }=1 \\
\text { where: GWP = Gray Water Reusing; } \\
\text { TWW = Total Water Washbasin }\end{array}$ \\
\hline 33 & WtMs & Rain water reusing $[17,16]$ & $\begin{array}{c}\text { RWR/TRW = 1 } \\
\text { where: } \mathrm{RWP}=\text { Rain Water Reusing } \\
\text { TRW = Total Rain Water }\end{array}$ & $\begin{array}{c}\mathrm{RWR} / \mathrm{TRW}=1 \\
\text { where: } \mathrm{RWP}=\text { Rain Water Reusing } \\
\text { TRW = Total Rain Water }\end{array}$ & $\begin{array}{c}\mathrm{RWR} / \mathrm{TRW}=1 \\
\text { where: } \mathrm{RWP}=\text { Rain Water Reusing } \\
\text { TRW }=\text { Total Rain Water }\end{array}$ \\
\hline
\end{tabular}

Table 4 Environmental protection and quality of the indoor key performance indicators

\begin{tabular}{|c|c|c|c|c|}
\hline \multirow{2}{*}{ No. } & \multicolumn{2}{|c|}{ Category } & \multicolumn{2}{|c|}{ Key Performance Indicators } \\
\cline { 2 - 5 } & \multirow{2}{*}{ Code } & Goals & \multicolumn{2}{|c|}{ Environmental Protection and Quality of the indoor (EPQ) } \\
\cline { 3 - 5 } 1 & SDS & $\begin{array}{c}\text { Environmental Sustainability } \\
\text { Strategy [15] }\end{array}$ & Environmental KPI & Economic KPI \\
\hline
\end{tabular}




\begin{tabular}{|c|c|c|c|c|c|}
\hline \multirow{3}{*}{ No. } & \multicolumn{2}{|r|}{ Category } & \multicolumn{3}{|c|}{ Key Performance Indicators } \\
\hline & \multirow{2}{*}{ Code } & \multirow{2}{*}{ Goals } & \multicolumn{3}{|c|}{ Environmental Protection and Quality of the indoor (EPQ) } \\
\hline & & & Environmental KPI & \begin{tabular}{|c|} 
Economic KPI \\
\end{tabular} & Social KPI \\
\hline 2 & EMS & $\begin{array}{l}\text { Environmental Management } \\
\text { System }\end{array}$ & \multicolumn{3}{|c|}{$\begin{array}{c}\qquad \mathrm{EMS}=1 \\
\text { where: } \mathrm{EMS}=\text { Environmental Management System }\end{array}$} \\
\hline 3 & EMS & $\begin{array}{c}\text { Externally verified } \\
\text { environmental management } \\
\text { system (EMS) in the last five } \\
\text { years? [15] } \\
\end{array}$ & $\mathrm{x}$ & $\mathrm{x}$ & $\begin{array}{l}\mathrm{AFYV}=1 \\
\text { where: AFYV }=\text { At least a Five-Year } \\
\text { Verification }\end{array}$ \\
\hline 4 & EMs & $\begin{array}{l}\text { Low-carbon energy use } \\
{[14]}\end{array}$ & $\begin{array}{c}\text { LCE/TEU = } 1 \\
\text { where: LCE = Low carbon energy; } \\
\text { TEU = Total Energy Usage }[21,20]\end{array}$ & $\mathrm{x}$ & $\mathrm{x}$ \\
\hline 5 & EMs & $\begin{array}{c}\text { Number of renewable energy } \\
\text { sources in } \\
\text { HEB [13] }\end{array}$ & $\begin{array}{c}\text { RES/TES=1 } \\
\text { where: RES = Renewable Energy } \\
\quad \text { Sources } \\
\text { TES=Total Energy Sources }[21,20]\end{array}$ & $\mathrm{x}$ & $\mathrm{x}$ \\
\hline 6 & EMs & $\begin{array}{l}\text { The ratio of renewable energy } \\
\text { produced towards energy } \\
\text { HEB's usage [13] }\end{array}$ & $\begin{array}{c}\mathrm{RE} / \mathrm{TEU}=1 \\
\text { where: } \mathrm{RES}=\text { Ratio of Renewable } \\
\text { Energy } \\
\text { TEU = Total Energy Usage }[21,20]\end{array}$ & $\mathrm{x}$ & $\mathrm{x}$ \\
\hline 7 & EMs & $\begin{array}{c}\text { Percentage of renewable energy } \\
\text { generated onsite or off site } \\
\text { compared to consumption of } \\
\text { grid electricity [15] }\end{array}$ & $\begin{array}{c}\text { REG/TEU }=1 \\
\text { where: } \mathrm{REG}=\text { Renewable Energy } \\
\text { Generated } \\
\text { TEU }=\text { Total Energy Usage }[21,20]\end{array}$ & $\mathrm{x}$ & $\mathrm{x}$ \\
\hline 8 & EMs & $\begin{array}{c}\text { Total percentage of renewable } \\
\text { energy purchased through green } \\
\text { tariffs }[15]\end{array}$ & $\begin{array}{c}\text { TREP/TEP }=1 \\
\text { where: } \text { TREP = Total renewable } \\
\text { energy purchase } \\
\text { TEP = Total Energy Purchase }[21,20]\end{array}$ & $\begin{array}{c}\text { TREP/TEP }=1 \\
\text { where: } \begin{array}{c}\text { TREP }=\text { Total renewable } \\
\text { energy purchase } \\
\text { TEP = Total Energy Purchase }[21,20]\end{array} \\
\end{array}$ & $\mathrm{x}$ \\
\hline 9 & EMs & $\begin{array}{c}\text { Clean and renewable energy } \\
{[17,16,14]}\end{array}$ & $\begin{array}{c}\mathrm{CRE} / \mathrm{EU}=1 \\
\text { where: } \mathrm{CRE}=\text { Clean and Renewable } \\
\text { Energy } \\
\mathrm{EU}=\text { Energy Usage }[21,20]\end{array}$ & $\begin{array}{c}\mathrm{CRE} / \mathrm{EU}=1 \\
\text { where: } \mathrm{CRE}=\text { Clean and Renewable } \\
\quad \text { Energy } \\
\mathrm{EU}=\text { Energy Usage }[21,20]\end{array}$ & $\mathrm{x}$ \\
\hline
\end{tabular}




\begin{tabular}{|c|c|c|c|c|c|}
\hline \multirow{3}{*}{ No. } & \multicolumn{2}{|r|}{ Category } & \multicolumn{3}{|c|}{$\begin{array}{c}\text { Key Performance Indicators } \\
\text { Fnyironmental Protection and Oplity of the indoor (FPO) }\end{array}$} \\
\hline & \multirow{2}{*}{ Code } & \multirow{2}{*}{ Goals } & \multicolumn{3}{|c|}{ Environmental Protection and Quality of the indoor (EPQ) } \\
\hline & & & Environmental KPI & Economic KPI & Social KPI \\
\hline 10 & EMs & $\begin{array}{l}\text { Research on affordable and } \\
\text { clean energy [14]* }\end{array}$ & $\mathrm{x}$ & $\mathrm{x}$ & $\begin{array}{c}\text { RACE/TES = 1 } \\
\text { where: } \text { RACE = Research on } \\
\text { Affordable and Clean Energy; } \\
\text { TES = Total specializations }\end{array}$ \\
\hline 11 & ESM & $\begin{array}{c}\text { Environmental education } \\
\text { measures (students) } \\
{[14]}\end{array}$ & $\mathrm{x}$ & $\mathrm{x}$ & $\begin{array}{c}\mathrm{MET}=1 \\
\text { where: } \mathrm{MET}=\text { Monthly periodic } \\
\text { Environmental Training }\end{array}$ \\
\hline 12 & EMS & $\begin{array}{c}\text { Environmental education } \\
\text { measures (staff) } \\
{[14]}\end{array}$ & & & $\begin{array}{c}\mathrm{MET}=1 \\
\text { where: } \mathrm{MET}=\text { Monthly periodic } \\
\text { Environmental Training }\end{array}$ \\
\hline 13 & GGMs & $\begin{array}{c}\text { Greenhouse Gasses } \\
\text { Management system } \\
{[13,14,15,16,17]}\end{array}$ & \multicolumn{3}{|c|}{$\begin{array}{c}\mathrm{CMs}=1 \\
\text { where: } \\
\text { rbon Manager }\end{array}$} \\
\hline 14 & GGMs & $\begin{array}{c}\text { Greenhouse gas emission } \\
\text { reductions program }[13,14,17]\end{array}$ & $\begin{array}{c}\text { GERP }=1 \\
\begin{array}{c}\text { GERP }=\text { Greenhouse gas Emission } \\
\text { Reductions Program }\end{array}\end{array}$ & $\begin{array}{c}\text { GERP }=1 \\
\begin{array}{c}\text { GERP }=\text { Greenhouse gas Emission } \\
\text { Reductions Program }\end{array} \\
\end{array}$ & $\begin{array}{c}\text { GERP }=1 \\
\begin{array}{c}\text { GERP }=\text { Greenhouse gas Emission } \\
\text { Reductions Program }\end{array}\end{array}$ \\
\hline 15 & WtMs & $\begin{array}{c}\text { Ratio of research on clean water } \\
\text { and sanitation towards total } \\
\text { research [14]* }\end{array}$ & ( & (1) & $\begin{array}{c}\text { RCWS/TSST }=1 \\
\text { where: } \text { RCWS = research on clean } \\
\text { water and sanitation; } \\
\text { RRCP = Research on Responsible } \\
\text { Consuption and Production; } \\
\text { TSST = Total Specializations that } \\
\text { Study the Topic } \\
\end{array}$ \\
\hline 16 & WtMs & \begin{tabular}{|c|} 
Strategy implemented for \\
responsible water consumption \\
in the HEB community [14, 13, \\
$15]$
\end{tabular} & $\mathrm{x}$ & $\mathrm{x}$ & $\begin{array}{c}\text { SIRWCC }=1 \\
\text { where: } \text { SIRWCC }=\text { Strategy } \\
\text { Implemented for Responsible Water } \\
\text { Consumption in the HEB Community }\end{array}$ \\
\hline
\end{tabular}


Table 5 Environmental protection and quality of the indoor key performance indicators

\begin{tabular}{|c|c|c|c|c|c|}
\hline \multirow{3}{*}{ No. } & \multicolumn{2}{|r|}{ Category } & \multirow{2}{*}{\multicolumn{3}{|c|}{$\begin{array}{c}\text { Key Performance Indicators } \\
\text { Human Resoure and }\end{array}$}} \\
\hline & \multirow{2}{*}{ Code } & \multirow{2}{*}{ Goals } & & & \\
\hline & & & Environmental KPI & Economic KPI & Social KPI \\
\hline 1 & GEC & $\begin{array}{l}\text { Accessibility and inclusive of } \\
\text { first-generation students } \\
\text { [14] }\end{array}$ & $\mathrm{x}$ & $\mathrm{x}$ & $\begin{array}{c}\text { IAIR/TAIR }=1 \\
\text { where: IAIR = Implemented } \\
\text { Accessibility and Inclusion } \\
\text { Regulations; } \\
\text { TAIR = Total Accessibility and } \\
\text { Inclusion Regulations }\end{array}$ \\
\hline 2 & GEC & $\begin{array}{l}\text { Disability student access } \\
\text { measures implemented } \\
\text { [14] }\end{array}$ & $\mathrm{x}$ & $\mathrm{x}$ & $\begin{array}{c}\text { DAMI/DAM =1 } \\
\text { where: } \text { DAMI = Disability Access } \\
\text { Measures Implemented; } \\
\text { DAM = Disability Access Measures }\end{array}$ \\
\hline 3 & GEC & $\begin{array}{l}\text { Minority student access } \\
\text { measures implement } \\
{[14]}\end{array}$ & $\mathrm{x}$ & $\mathrm{x}$ & $\begin{array}{c}\text { MAMI/MAM = 1 } \\
\text { where: } \text { MAMI = Minority access } \\
\text { measures implemented; } \\
\text { MAM = Minority access measures }\end{array}$ \\
\hline 4 & GEC & $\begin{array}{c}\text { Proportion of senior female } \\
\text { academics [14] }\end{array}$ & $\mathrm{x}$ & $\mathrm{x}$ & $\begin{array}{c}\text { SFA/SMA } \approx 1 \\
\text { where: SFA = Senior female academic; } \\
\text { SMA = Senior male academic }\end{array}$ \\
\hline 5 & GEC & $\begin{array}{c}\text { Proportion of women receiving } \\
\text { degrees D }\end{array}$ & $\mathrm{x}$ & $\mathrm{x}$ & $\begin{array}{c}\mathrm{FG} / \mathrm{MS} \approx 1 \\
\text { where: } \mathrm{FG}=\text { Female graduates; } \\
\mathrm{MG}=\text { Male graduates }\end{array}$ \\
\hline 6 & GEC & $\begin{array}{c}\text { Research on reducing } \\
\text { inequalities } \\
{[14]}\end{array}$ & $\mathrm{x}$ & $\mathrm{x}$ & $\begin{array}{c}\text { RRI/TS=1 } \\
\text { where: } \mathrm{RRI}=\text { Research on Reducing } \\
\text { Inequalities; } \\
\text { TS = Total specializations }\end{array}$ \\
\hline 7 & GEC & $\begin{array}{l}\text { Students from developing } \\
\text { countries [14] }\end{array}$ & $\mathrm{x}$ & $\mathrm{x}$ & $\begin{array}{c}\mathrm{UCS} / \mathrm{DCS}=1 \\
\text { where: } \mathrm{UCS}=\text { Underdeveloped } \\
\text { country students; } \\
\text { DCS = Developed country students }\end{array}$ \\
\hline
\end{tabular}




\begin{tabular}{|c|c|c|c|c|c|}
\hline \multirow{3}{*}{ No. } & \multicolumn{2}{|r|}{ Category } & \multirow{2}{*}{\multicolumn{3}{|c|}{$\begin{array}{c}\text { Key Performance Indicators } \\
\text { Human Resource and other Stakeholders (HRS) }\end{array}$}} \\
\hline & \multirow{2}{*}{ Code } & \multirow{2}{*}{ Goals } & & & \\
\hline & & & Environmental KPI & Economic KPI & Social KPI \\
\hline 8 & GEC & $\begin{array}{c}\text { Female students with } \\
\text { disabilitiess } \\
{[14]} \\
\end{array}$ & 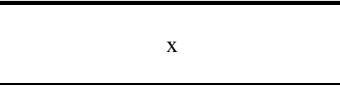 & $\mathrm{x}$ & $\begin{array}{l}\text { FDS }=1 \\
\text { where: FDS = Female student with } \\
\text { Disabilities per Specialization [30] }\end{array}$ \\
\hline 9 & GEC & $\begin{array}{c}\text { Male students with disabilitiess } \\
{[14]}\end{array}$ & $\mathrm{x}$ & $\mathrm{x}$ & $\begin{array}{c}\text { MDS = 1 } \\
\text { where: MDS = Male student with } \\
\text { Disabilities per Specialization [30] }\end{array}$ \\
\hline 10 & GEC & $\begin{array}{l}\text { Female staff with disabilities } \\
\text { [14] }\end{array}$ & $\mathrm{x}$ & $\begin{array}{c}\text { FDD }=1 \\
\text { where: FDD = Female staff with } \\
\text { disabilities per department }[30]\end{array}$ & $\begin{array}{c}\text { FDD }=1 \\
\text { where: FDD }=\text { Female staff with } \\
\text { disabilities per department }[30]\end{array}$ \\
\hline 11 & GEC & $\begin{array}{l}\text { Male staff with disabilities } \\
{[14]}\end{array}$ & $\mathrm{x}$ & $\begin{array}{c}\text { MDD }=1 \\
\text { where: MDD = Male staff with } \\
\text { disabilities per department [30] }\end{array}$ & $\begin{array}{c}\text { MDD }=1 \\
\text { where: } \mathrm{MDD}=\text { Male staff with } \\
\text { disabilities per department [30] }\end{array}$ \\
\hline 12 & GEC & $\begin{array}{c}\text { Measures against } \\
\text { discrimination implemented } \\
{[14]}\end{array}$ & $\mathrm{x}$ & 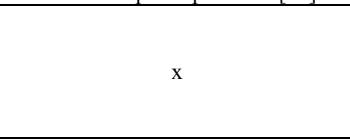 & $\begin{array}{c}\text { MADI/TMAD }=1 \\
\text { where: } \text { MADI = Measures Against } \\
\text { Discrimination Implemented; } \\
\text { TMAD = Total Measures Against } \\
\text { Discrimination }\end{array}$ \\
\hline 13 & SDS & $\begin{array}{l}\text { Public sustainability policy } \\
\qquad[15]\end{array}$ & $\begin{array}{c}\text { PSP }=1 \\
\begin{array}{c}\text { where: PSP }= \\
\text { Public sustainability } \\
\text { policy }\end{array}\end{array}$ & 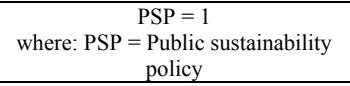 & $\begin{array}{c}\text { PSP }=1 \\
\begin{array}{c}\text { where: } \text { PSP }=\text { Public sustainability } \\
\text { policy }\end{array}\end{array}$ \\
\hline 14 & SDS & $\begin{array}{c}\text { Chapters regarding } \\
\text { sustainability in public policies } \\
{[15]}\end{array}$ & $\begin{array}{c}\mathrm{CS} / \mathrm{TPP}=1 \\
\text { where: } \mathrm{CS}=\text { Chapters regarding } \\
\text { Sustainability; } \\
\text { TPP = Total Public Policy }\end{array}$ & $\begin{array}{c}\mathrm{CS} / \mathrm{TPP}=1 \\
\text { where: } \mathrm{CS}=\text { Chapters regarding } \\
\text { Sustainability; } \\
\text { TPP = Total Public Policy }\end{array}$ & $\begin{array}{c}\mathrm{CS} / \mathrm{TPP}=1 \\
\text { where: } \mathrm{CS}=\text { Chapters regarding } \\
\text { Sustainability; } \\
\text { TPP = Total Public Policy }\end{array}$ \\
\hline 15 & SDS & $\begin{array}{l}\text { Publication of a sustainability } \\
\text { report }[14,13]\end{array}$ & $\begin{array}{c}\mathrm{SR}=1 \\
\text { where: } \mathrm{SR}=\text { Sustainability Report; }\end{array}$ & $\begin{array}{c}\mathrm{SR}=1 \\
\text { where: } \mathrm{SR}=\text { Sustainability Report; }\end{array}$ & $\begin{array}{c}\mathrm{SR}=1 \\
\text { where: } \mathrm{SR}=\text { Sustainability Report; }\end{array}$ \\
\hline 16 & SDS & $\begin{array}{c}\text { Chapter regarding } \\
\text { sustainability in publication } \\
\text { reports [14] }\end{array}$ & $\begin{array}{c}\mathrm{CS} / \mathrm{TR}=1 \\
\text { where: } \mathrm{CS}=\mathrm{Chapter} \text { regarding } \\
\text { Sustainability; } \mathrm{TR}=\text { total reports }\end{array}$ & $\begin{array}{c}\mathrm{CS} / \mathrm{TR}=1 \\
\text { where: } \mathrm{CS}=\mathrm{Chapter} \text { regarding } \\
\text { Sustainability; } \mathrm{TR}=\text { total reports }\end{array}$ & $\begin{array}{c}\mathrm{CS} / \mathrm{TR}=1 \\
\text { where: } \mathrm{CS}=\text { Chapter regarding } \\
\text { Sustainability; TR }=\text { total reports }\end{array}$ \\
\hline 17 & SDS & $\begin{array}{c}\text { Existence of a senior } \\
\text { management member with }\end{array}$ & $\begin{array}{c}\text { SMMSR }=1 \\
\text { where: } \text { SMMSR }= \\
\end{array}$ & $\begin{array}{c}\text { SMMSR }=1 \\
\text { where: } \mathrm{SMMSR}=\end{array}$ & $\begin{array}{c}\text { SMMSR }=1 \\
\text { where: } \mathrm{SMMSR}=\end{array}$ \\
\hline
\end{tabular}




\begin{tabular}{|c|c|c|c|c|c|}
\hline \multirow{3}{*}{ No. } & \multicolumn{2}{|r|}{ Category } & \multicolumn{3}{|c|}{ Key Performance Indicators } \\
\hline & \multirow{2}{*}{ Code } & \multirow{2}{*}{ Goals } & \multicolumn{3}{|c|}{ Human Resource and other Stakeholders (HRS) } \\
\hline & & & Environmental KPI & \begin{tabular}{|c|} 
Economic KPI \\
\end{tabular} & Social KPI \\
\hline & & sustainability responsibilities & $\begin{array}{l}\text { Senior Management Member with } \\
\text { Sustainability Responsibilities }\end{array}$ & $\begin{array}{l}\text { Senior Management Member with } \\
\text { Sustainability Responsibilities }\end{array}$ & $\begin{array}{l}\text { Senior Management Member with } \\
\text { Sustainability Responsibilities }\end{array}$ \\
\hline 18 & SDS & $\begin{array}{l}\text { Existence of people from the } \\
\text { management staff with [15] } \\
\text { dedicated sustainability role }\end{array}$ & $\begin{array}{c}\text { PSR }=1 \\
\text { where: PSR }=\text { People with dedicated } \\
\text { Sustainability Role }\end{array}$ & $\begin{array}{c}\text { PSR }=1 \\
\text { where: PSR }=\text { People with dedicated } \\
\text { Sustainability Role }\end{array}$ & $\begin{array}{c}\text { PSR }=1 \\
\text { where: } \text { PSR }=\text { People with dedicated } \\
\text { Sustainability Role }\end{array}$ \\
\hline 19 & SDS & $\begin{array}{l}\text { Staff are supported with a } \\
\text { budget for sustainability [15] }\end{array}$ & 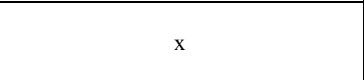 & $\begin{array}{c}\mathrm{SDB}=1 \\
\begin{array}{c}\text { where: } \mathrm{SDB}=\text { Sustainability } \\
\text { dedicated budget }\end{array}\end{array}$ & $\begin{array}{c}\text { SDB }=1 \\
\text { where: } \mathrm{SDB}=\begin{array}{c}\text { Sustainability dedicated } \\
\text { budget }\end{array}\end{array}$ \\
\hline 20 & SDS & $\begin{array}{l}\text { All staff involvement in } \\
\text { sustainability [15] }\end{array}$ & $\begin{array}{c}\text { IMS/TSM }=1 \\
\text { where: IMS = Involved Member Staff } \\
\text { TSM = Total Staff Member }\end{array}$ & $\begin{array}{c}\text { IMS/TSM =1 } \\
\text { where: IMS = Involved Member Staff } \\
\text { TSM = Total Staff Member }\end{array}$ & $\begin{array}{c}\text { IMS/TSM }=1 \\
\text { where: IMS = Involved Member Staff } \\
\text { TSM = Total Staff Member }\end{array}$ \\
\hline 21 & SDS & $\begin{array}{l}\text { A strategy for student and staff } \\
\text { engagement in sustainable } \\
\text { development [15] }\end{array}$ & $\begin{array}{c}\text { SSSESD = 1 } \\
\text { where: SSSESD }=\text { strategy for student } \\
\text { and staff engagement in sustainable } \\
\text { development }\end{array}$ & $\begin{array}{c}\text { SSSESD }=1 \\
\text { where: } \mathrm{SSSESD}=\text { strategy for student } \\
\text { and staff engagement in sustainable } \\
\text { development }\end{array}$ & $\begin{array}{c}\text { SSSESD }=1 \\
\begin{array}{c}\text { where: } \mathrm{SSSESD}=\text { strategy for student } \\
\text { and staff engagement in sustainable } \\
\text { development }\end{array}\end{array}$ \\
\hline 22 & SDS & $\begin{array}{c}\text { Sustainable development goals } \\
\text { published (SDG) report [14] }\end{array}$ & $\begin{array}{c}\text { SDGPR }=1 \\
\text { where: Sustainable Development } \\
\text { Goals Published Report }\end{array}$ & $\begin{array}{c}\text { SDGPR }=1 \\
\text { where: Sustainable Development } \\
\text { Goals Published Report }\end{array}$ & $\begin{array}{c}\text { SDGPR }=1 \\
\text { where: Sustainable Development Goals } \\
\text { Published Report }\end{array}$ \\
\hline 23 & SDS & $\begin{array}{l}\text { Chapters regarding SDG in } \\
\text { public policies [14] }\end{array}$ & $\begin{array}{c}\mathrm{CSDG} / \mathrm{TPP}=1 \\
\text { where: } \mathrm{CSDG}=\text { Chapter regarding } \\
\text { Sustainable Development Goals; } \\
\text { TPP = Total Public Policies }\end{array}$ & $\begin{array}{c}\mathrm{CSDG} / \mathrm{TPP}=1 \\
\text { where: } \mathrm{CSDG}=\text { Chapter regarding } \\
\text { Sustainable Development Goals; } \\
\text { TPP = Total Public Policies }\end{array}$ & $\begin{array}{c}\mathrm{CSDG} / \mathrm{TPP}=1 \\
\text { where: } \mathrm{CSDG}=\text { Chapter regarding } \\
\text { Sustainable Development Goals; } \\
\text { TPP = Total Public Policies }\end{array}$ \\
\hline 24 & SDS & $\begin{array}{l}\text { The student representation on } \\
\text { committees concerned with } \\
\text { estates, planning, finance and } \\
\text { strategic decision making [15] }\end{array}$ & $\mathrm{x}$ & $\begin{array}{c}\text { SRI }=1 \\
\text { where: } \text { SRI }=\text { Student Representation } \\
\text { Involvement }\end{array}$ & $\begin{array}{cc}\text { SRI }=1 \\
\text { where: SRI }=\text { Student Representation } \\
\text { Involvement }\end{array}$ \\
\hline 25 & SDS & $\begin{array}{c}\text { The ratio of sustainability } \\
\text { chapters towards total courses } \\
{[13,15]^{*}}\end{array}$ & $\begin{array}{c}\mathrm{SC} / \mathrm{TC}=1 \\
\text { where: } \mathrm{SC}=\text { sustainability chapters; } \\
\mathrm{TC}=\text { total courses }\end{array}$ & $\begin{array}{c}\mathrm{SC} / \mathrm{TC}=1 \\
\text { where: } \mathrm{SC}=\text { sustainability chapters; } \\
\mathrm{TC}=\text { total courses }\end{array}$ & $\begin{array}{c}\mathrm{SC} / \mathrm{TC}=1 \\
\text { where: } \mathrm{SC}=\text { sustainability chapters; } \\
\mathrm{TC}=\text { total courses }\end{array}$ \\
\hline 26 & SDS & $\begin{array}{c}\text { Sustainability dedicated course } \\
{[13,15]}\end{array}$ & $\begin{array}{c}\text { SDC }=1 \\
\text { where: } \mathrm{SDC}=\text { Sustainability }\end{array}$ & $\begin{array}{c}\text { SDC }=1 \\
\text { where: } \mathrm{SDC}=\text { Sustainability }\end{array}$ & $\begin{array}{c}\text { SDC }=1 \\
\text { where: } \text { SDC }=\text { Sustainability Dedicated }\end{array}$ \\
\hline
\end{tabular}




\begin{tabular}{|c|c|c|c|c|c|}
\hline \multirow{3}{*}{ No. } & \multicolumn{2}{|r|}{ Category } & \multirow{2}{*}{\multicolumn{3}{|c|}{$\begin{array}{c}\text { Key Performance Indicators } \\
\text { Human Resource and other Stakeholders (HRS) }\end{array}$}} \\
\hline & \multirow{2}{*}{ Code } & \multirow{2}{*}{ Goals } & & & \\
\hline & & & Environmental KPI & \begin{tabular}{|c|} 
Economic KPI \\
\end{tabular} & Social KPI \\
\hline & & & Dedicated Course & Dedicated Course & Course \\
\hline 27 & SDS & $\begin{array}{l}\text { Sustainability dedicated } \\
\text { specialization }[13,15]^{*}\end{array}$ & $\begin{array}{c}\text { SDS }=1 \\
\text { where: } \text { SDS }=\text { Sustainability dedicated } \\
\text { specialization }\end{array}$ & $\begin{array}{c}\text { SDS = 1 } \\
\text { where: } \text { SDS = Sustainability } \\
\text { dedicated specialization }\end{array}$ & $\begin{array}{c}\text { SDS }=1 \\
\text { where: } \mathrm{SDS}=\text { Sustainability dedicated } \\
\text { specialization }\end{array}$ \\
\hline 28 & SDS & $\begin{array}{l}\text { The ratio of sustainability } \\
\text { research funding towards total } \\
\text { research [13]* }\end{array}$ & 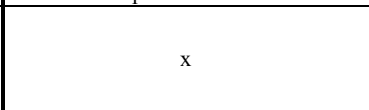 & ${ }^{2}$ & $\begin{array}{c}\text { RSR } / \mathrm{TS}=1 \\
\text { where: } \mathrm{RSR}=\text { Ratio of Sustainability } \\
\text { Research; } \\
\mathrm{TS}=\text { Total specializations }\end{array}$ \\
\hline 29 & SDS & $\begin{array}{l}\text { Annual number of scholarly } \\
\text { events related to environment } \\
\text { and sustainability }[13,14]\end{array}$ & $\begin{array}{c}\text { ASEES }=1 \\
\text { where: ASEES = Annual Scholarly } \\
\text { Events related to Environment and } \\
\text { Sustainability } \\
\end{array}$ & $\begin{array}{c}\text { RSR/TS }=1 \\
\text { where: } \mathrm{RSR}=\text { Ratio of Sustainability } \\
\text { Research; } \\
\text { TS = Total specializations }\end{array}$ & $\begin{array}{c}\text { RSR } / \mathrm{TS}=1 \\
\text { where: RSR }=\text { Ratio of Sustainability } \\
\text { Research; } \\
\mathrm{TS}=\text { Total specializations }\end{array}$ \\
\hline 30 & SDS & $\begin{array}{l}\text { Number of student } \\
\text { organizations related to } \\
\text { environment and } \\
\text { sustainability* [13] } \\
\end{array}$ & $\begin{array}{c}\text { NSOES }=1 \\
\text { where: Number of Student } \\
\text { Organizations Related to Environment } \\
\text { and Sustainability }\end{array}$ & 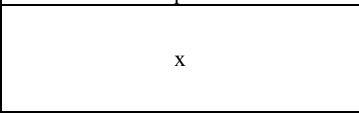 & $\begin{array}{c}\text { NSOES }=1 \\
\text { where: Number of Student } \\
\text { Organizations Related to Environment } \\
\text { and Sustainability }\end{array}$ \\
\hline 31 & SDS & $\begin{array}{l}\text { Existence of a HEB-run } \\
\text { sustainability website [13] }\end{array}$ & 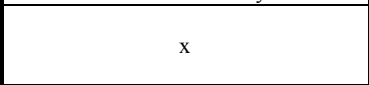 & $\begin{array}{c}\text { HEBSW }=1 \\
\text { where: } \text { HEBSW }=\text { HEB-run } \\
\text { sustainable website }\end{array}$ & $\begin{array}{c}\text { HEBSW }=1 \\
\text { where: HEBSW }=\text { HEB-run } \\
\text { sustainable website }\end{array}$ \\
\hline 32 & SDS & $\begin{array}{l}\text { Lifelong learning measures } \\
\text { implemented [14] }\end{array}$ & $\mathrm{x}$ & 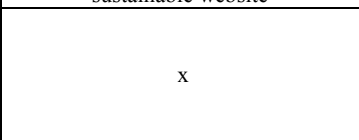 & $\begin{array}{c}\text { LLMI/TLLM = } 1 \\
\text { where: LLMI = Lifelong Learning } \\
\text { Measures Implemented; } \\
\text { TLLM = Total Lifelong Learning } \\
\text { Measures }\end{array}$ \\
\hline 33 & SDS & $\begin{array}{l}\text { Implemented strategy for } \\
\text { sustainable development [15] }\end{array}$ & $\begin{array}{c}\text { ISSD }=1 \\
\text { where: ISSD = Implemented Strategy } \\
\text { for Sustainable Development }\end{array}$ & $\begin{array}{c}\text { ISSD }=1 \\
\text { where: ISSD = Implemented Strategy } \\
\text { for Sustainable Development }\end{array}$ & $\begin{array}{c}\text { ISSD }=1 \\
\text { where: ISSD = Implemented Strategy } \\
\text { for Sustainable Development }\end{array}$ \\
\hline 34 & SDS & $\begin{array}{l}\text { Implemented education on the } \\
\text { SDGs [14] }\end{array}$ & $\begin{array}{c}\text { IESDG = 1 } \\
\text { where: IESDG = Implemented } \\
\text { education on the SDGs }\end{array}$ & $\begin{array}{c}\text { IESDG }=1 \\
\text { where: IESDG = Implemented } \\
\text { education on the SDGs }\end{array}$ & $\begin{array}{c}\text { IESDG }=1 \\
\text { where: } \text { IESDG }=\text { Implemented } \\
\text { education on the SDGs }\end{array}$ \\
\hline
\end{tabular}




\begin{tabular}{|c|c|c|c|c|c|}
\hline \multirow{3}{*}{ No. } & \multicolumn{2}{|r|}{ Category } & \multicolumn{3}{|c|}{ Key Performance Indicators } \\
\hline & \multirow{2}{*}{ Code } & \multirow{2}{*}{ Goals } & \multicolumn{3}{|c|}{ Human Resource and other Stakeholders (HRS) } \\
\hline & & & Environmental KPI & Economic KPI & Social KPI \\
\hline 35 & SDS & $\begin{array}{l}\text { The ratio of SDG research } \\
\text { towards total research }\end{array}$ & $\begin{array}{c}\text { SDGR/TSST = 1 } \\
\text { where: } \text { SDGR = Ratio of SDG } \\
\text { Research; } \\
\text { TSST = Total Specializations that } \\
\text { Study the Topic }\end{array}$ & $\mathrm{x}$ & $\begin{array}{c}\text { SDGR/TSST }=1 \\
\text { where: } \text { SDGR = Ratio of SDG } \\
\text { Research; } \\
\text { TSST = Total Specializations that } \\
\text { Study the Topic }\end{array}$ \\
\hline 36 & SDS & $\begin{array}{c}\text { Research on responsible } \\
\text { consumption and production } \\
{[14]^{*}}\end{array}$ & $\begin{array}{c}\text { RRC/TSST =1 } \\
\text { where: RRCP = Research on } \\
\text { Responsible Consumption and } \\
\text { Production; } \\
\text { TSST = Total Specializations that } \\
\text { Study the Topic }\end{array}$ & $\begin{array}{c}\text { RRC/TSST }=1 \\
\text { where: RRCP = Research on } \\
\text { Responsible Consumption and } \\
\text { Production; } \\
\text { TSST }=\text { Total Specializations that } \\
\text { Study the Topic }\end{array}$ & $\begin{array}{c}\text { RRC/TSST }=1 \\
\text { where: } \text { RRCP }=\text { Research on } \\
\text { Responsible Consumption and } \\
\text { Production; } \\
\text { TSST }=\text { Total Specializations that } \\
\text { Study the Topic }\end{array}$ \\
\hline
\end{tabular}

There are three cases to consider when calculating the identified KPIs:

- Case I: most KPIs were performed in relation to the result in the range $0-1$, where 1 is considered ideal.

- Case II: there are performance indicators that could not be expressed in the form of a report. Thus, in order to facilitate the calculations, the evaluation of the sustainability of an HEB will take into account the fulfillment of the criterion (grade with 1) or the non-fulfillment (grade with 0). If two or more HEBs are compared, they will be separated according to the performance obtained. For example, in the case of the "staff with sustainability role" criterion, if one HEB has one employee and the other has three, both will meet the criterion, but the one with three employees will win because 3 will represent $100 \%$ (1) and 1 will represent only $33.33 \%(0.33)$.

- Case III: There are some particular criteria that are taken into account only in the HEBs for which they are addressed, marked with "*". For example, the criterion "Research on responsible consumption and production" which could not be applied in a HEB with a field of humanistic study.

For the calculation of KPIs, a mathematical model was developed (formula 3.1), based on mathematical models studied in the consulted references [35 - 37]. This is a linear regression formula that calculates the weighted sum of all the KPIs of the three main categories, the result being in the range [0-1], where 1 is ideal. 


$$
M_{H E B}=\left(\sum_{i=1}^{n} B_{i}\right) * P 1+\left(\sum_{j=1}^{m} E_{j}\right) * P 2+\left(\sum_{k=1}^{o} H_{o}\right) * P 3=1
$$

where:

- $\mathrm{B}=\mathrm{BDM}$

- $\mathrm{E}=\mathrm{EPQ}$

- $\mathrm{H}=\mathrm{HRS}$

- $\mathrm{n}=$ total number of BDM KPIs;

- $\mathrm{m}=$ total number of EPQ KPIs;

- $\mathrm{o}=$ total number of HRS KPIs;

- $\mathrm{P} 1=\mathrm{BDM}$ weight;

- $\mathrm{P} 2$ = EPQ weight;

- $\mathrm{P} 3=$ HRS weight.

$$
\begin{gathered}
P 1=P 2=P 3=33,33 \% \\
P 1+P 2+P 3=100 \%
\end{gathered}
$$

In the proposed model all three Ps had a weight according to formula 3.2. However, this weighting may be chosen and different provided that it complies with formula 3.3.

Practically according to the proposed HEB model, it should obtain a performance score in the range of $0.75-1.00$, in order to be considered a sustainable building.

\section{Conclusions}

\subsection{Discutions}

Following this study, it was possible to develop a model that can facilitate the integration of sustainability in HEBs. At the same time, the proposed model can be used to evaluate the sustainability performance of a HEB or even to compare two or more HEBs.

Another important aspect is that the designed model is a modular one. Each module is represented by a category of criteria that evaluate from the perspective of the three pillars of sustainability. With the help of these modules it is possible to independently assess the level of building sustainability, environmental protection or human resources.

The model contains 85 evaluation criteria that have been transformed into specific KPIs for evaluating the sustainability performance of an HEB. The 85 criteria were grouped into three categories (modules) and 10 subcategories. Of these, only the SDS subcategory is found in all three modules, reaching the three pillars of sustainability in each module.

The proposed mathematical model calculates the weighted sum of the performance of each module to obtain an overview of the level of performance in terms of the sustainability of a HEB. For this model it was established that the weight of the three modules should be equal. However, this weighting may be chosen and different provided that it complies with formula 3.3 .

\subsection{Limitations}

The main limitation of the study is represented by the fact that the model is a conceptual one and it has not been implemented so far. Thus, it is proposed as future research directions to pilot the model on a HEB and compare it with other existing models.

Other limitations could be: the lack of experience of the first author, the relatively short time and the existing health situation. 
Acknowledgement: Project financed from Lucian Blaga University of Sibiu, The Research centre for sustainable products and processes \& Hasso Plattner Foundation research action LBUS-RRC-202001 .

\section{References}

1. C. Oprean, Managementul Calității Totale, (Sibiu, 2019)

2. Comisia Mondială pentru Mediu şi Dezvoltar, Viitorul nostru comun, (Oxford, Anglia, 1987)

3. F. Harris, Sustainable Development, Global Environmental Issues, (Marea Britanie, 2004)

4. A. Scott, Dimensions of Sustainability: architecture, form, technology, environment, Great Britain, 1998.

5. UICN, Strategia mondială de conservare: conservarea resurselor vii, (Glanda, Elveția, 1991)

6. J. Pronk, H. Mahbubul, Sustainable development: from concept to action, (New York, 1992)

7. A. I. Sârb, Contribuții privind managementul inovării în dezvoltarea clădirilor sustenabile, inteligente și incluzive, (Universitatea Tehnică din Cluj Napoca, Cluj Napoca, 2017)

8. https://www.greenofficemovement.org/sustainable-university/, [Accessed 14.3.2020]

9. V. Grecu, Contribuții privind sustenabilitatea în universități, (ULBS, Sibiu, 2012)

10. Y. Zhang, Z. Hu, R. Lin, J.-P. Zhang, Energy Reports, 7, 1326-1337 (2021)

11. J.-R. Lin, Automation in Construction, 103, 26-40 (2019)

12. ***, European Journal of Operational Research, 286, 309-320 (2020)

13. http://greenmetric.ui.ac.id/criteria-indicator/, [Accessed 14.4.2020]

14. https://www.timeshighereducation.com/impact-rankings-2020-methodology, [Accessed 14.8.2020]

15. https://peopleandplanet.org/university-league-methodology, [Accessed 14.4.2020]

16. $\mathrm{https://www.bestcolleges.com/features/greenest-universities/,} \mathrm{[Accessed} \mathrm{12.4.2020]}$

17. https://stars.aashe.org/resources-support/technical-manual/, [Accessed 12.4.2020]

18. https://eur-lex.europa.eu/legal-content/RO/TXT/?uri=LEGISSUM\%3Aen0021, [Accessed 123 2021]

19. https://eur-lex.europa.eu/legalcontent/EN/TXT/?uri=uriserv\%3AOJ.L .2018.156.01.0075.01.ENG, [Accessed 14.03.2021]

20. https://www.europarl.europa.eu/thinktank/en/document.html?reference=EPRS_BRI(202 0)659370, [Accessed 11.3.2021]

21. http://bpie.eu/wp-

content/uploads/2015/11/nZEB_Criteria_for_renovation_COHERENO.pdf, [Accessed 12.3.2021]

22. https://passivehouse.com/02 informations/02 passive-house-requirements/02 passivehouse-requirements.htm, [Accessed 12.3.2021]

23. https://www.proiect1.ro/servicii/pozitionare.html, [Accessed 12.03.2021]

24. http://legislatie.just.ro/Public/DetaliiDocumentAfis/227538, [Accessed 15.3.2021]

25. http://legislatie.just.ro/Public/DetaliiDocument/66970., [Accessed 16.3.2021]

26. UNESCO, Education for Sustainable Development Goals, (2017)

27. https://www.asro.ro/sr-en-iso-500012019-instrument-cheie-pentru-eficienta-energeticaa-organizatiilor/, [Accessed 12.3.2021]

28. https://recyclemania.org/participate/categories/race-zero-waste/, [Accessed 12.3.2021] 
29. https://scholarworks.umass.edu/cgi/viewcontent.cgi? article $=1003 \&$ context $=$ sustainableu mass studentshowcase, [Accessed 12.3.2021]

30. http://legislatie.just.ro/Public/DetaliiDocument/77815, [Accessed 12.3.2021

31. S. Attia, P. Alphonsine, M. Amer, G. Ruellan, Environmental and Sustainability Indicators, 7, p. 100052 (2020)

32. http://legislatie.just.ro/Public/DetaliiDocument/229693, [Accessed 14.03.2021]

33. https://www.euro.who.int/_ data/assets/pdf_file/0009/276624/School-environmentPolicies-current-statusen.pdf?fbclid=IwAR2ZZas1nXXG_h2uHdQx2C6wFc5ZPVzrMO9964J11qVda2LxtO XkaBLZyWc, [Accessed 28.3.2021]

34. https://www.sageglass.com/en/visionary-insights/first-european-standard-daylightbuildings, [Accessed 21.3.2021]

35. M.-V. Zerbes, Planificarea și proiectarea avansată a proceselor și produselor în industria auto (Techno Media, Sibiu, 2017)

36. L. Popescu, M. Zerbes, Measuring the performance of the manufacturing planning process. A conceptual model (Sibiu, 2018)

37. M.-V. Zerbes, L.-I. Cioca, V. Neamu, Developing a model of occupational health program (Sibiu, 2018) 